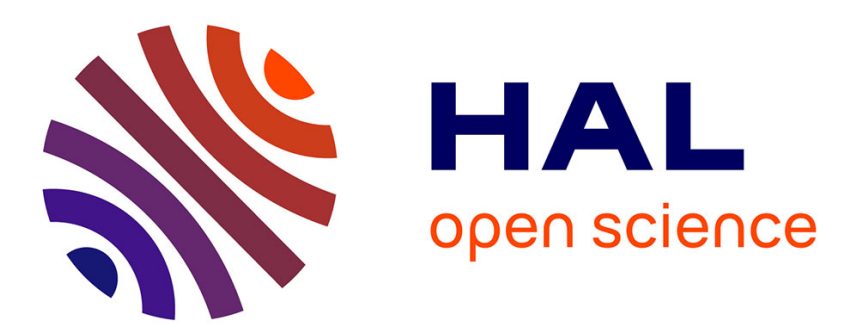

\title{
Topography-induced large-scale anti-parallel collective migration in vascular endothelium
}

Claire Leclech, David Gonzalez-Rodriguez, Aurélien Villedieu, Thévy Lok, Anne-Marie Déplanche, Abdul Barakat

\section{- To cite this version:}

Claire Leclech, David Gonzalez-Rodriguez, Aurélien Villedieu, Thévy Lok, Anne-Marie Déplanche, et al.. Topography-induced large-scale anti-parallel collective migration in vascular endothelium. 2021. hal-03456241

\section{HAL Id: hal-03456241 \\ https://hal.science/hal-03456241}

Preprint submitted on 30 Nov 2021

HAL is a multi-disciplinary open access archive for the deposit and dissemination of scientific research documents, whether they are published or not. The documents may come from teaching and research institutions in France or abroad, or from public or private research centers.
L'archive ouverte pluridisciplinaire HAL, est destinée au dépôt et à la diffusion de documents scientifiques de niveau recherche, publiés ou non, émanant des établissements d'enseignement et de recherche français ou étrangers, des laboratoires publics ou privés. 


\title{
Topography-induced large-scale anti-parallel collective
}

\section{migration in vascular endothelium}

\author{
Claire Leclech ${ }^{1}$, David Gonzalez-Rodriguez ${ }^{2}$, Aurélien Villedieu ${ }^{3}$, Thévy Lok ${ }^{1}$,
} Anne-Marie Déplanche ${ }^{4}$, Abdul I Barakat ${ }^{1 *}$

Affiliations:

${ }^{1}$ LadHyX, CNRS, Ecole Polytechnique, Institut Polytechnique de Paris, Palaiseau, France.

${ }^{2}$ Université de Lorraine, LCP-A2MC, F-57000, Metz, France.

${ }^{3}$ Institut Curie, PSL Research University, CNRS UMR 3215, INSERM U934, F-75248 Paris

Cedex 05, France; Sorbonne Universités, UPMC Univ Paris 06, CNRS, CNRS UMR 3215, INSERM U934, F-75005, France.

${ }^{4}$ University of Nantes, LS2N UMR 6004, 1 rue de la Nöe - BP 9210 Nantes 44321.

*Correspondence: Prof. Abdul I. Barakat - abdul.barakat@polytechnique.edu 


\begin{abstract}
Collective migration of vascular endothelial cells is central for embryonic development, angiogenesis, and wound closure. Although physical confinement of cell assemblies has been shown to elicit specific patterns of collective movement in various cell types, endothelial migration in vivo often occurs without confinement. Here we show that unconfined endothelial cell monolayers on microgrooved substrates that mimic the anisotropic organization of the extracellular matrix exhibit a new type of collective movement that takes the form of a periodic pattern of anti-parallel cell streams. We further establish that the development of these streams requires intact cell-cell junctions and that stream sizes are particularly sensitive to groove depth. Finally, we show that modeling the endothelial cell sheet as an active fluid with the microgrooves acting as constraints on cell orientation predicts the occurrence of the periodic anti-parallel cell streams as well as their lengths and widths. We posit that in unconfined cell assemblies, physical factors that constrain or bias cellular orientation such as anisotropic extracellular matrix cues or directed flow-derived shear forces dictate the pattern of collective cell movement.
\end{abstract}

\title{
Introduction
}

Collective cellular movement is central for many physiological and pathological processes including morphogenesis, angiogenesis, wound healing, and cancer invasion ${ }^{1}$. A prominent manifestation of collective cell behavior is the emergence of complex migration patterns whose structure and organization depend on many factors including the physical constraints to which the cells are subjected. For instance, meso-scale geometric confinement of cellular monolayers has been shown to organize otherwise chaotic cell movement into directed bidirectional motion, swirling, or even global rotation, with the specific pattern dependent on cell type and the size and shape of the confinement $\operatorname{area}^{2-7}$. 
Although geometric confinement is representative of some physiologically relevant scenarios, collective migration in vivo often occurs without such confinement. A prominent example is vascular endothelial cell wound healing following device- or disease-induced injury. In this case, the migrating cell sheets remain free of global confinement, but the cells' basal surfaces are nonetheless subjected to anisotropic biophysical cues due to the nano- to micro-scale topography of the thin fibers of the underlying extracellular matrix ${ }^{8,9}$. How these ubiquitous biophysical cues regulate collective cell migration patterns remains unknown. Here we address this question by studying the collective motion of vascular endothelial cells on anisotropic microgrooved substrates that constitute idealized mimics of extracellular matrix topography ${ }^{10}$. We show that unconfined endothelial monolayers on microgrooves exhibit a new type of collective migration that takes the form of periodic anti-parallel cell streams whose characteristic dimensions are considerably larger than either the groove or cell size, suggesting that these topographic constraints induce long-range effects. We also show that the emergence of the cell stream pattern requires intact cell-cell contacts and that the sizes of the streams are determined by the groove dimensions, most notably groove depth. Modeling the endothelial cell sheet as an active fluid with the microgrooves acting as constraints on cell orientation accurately predicts the occurrence of the periodic anti-parallel cell streams as well as their lengths and widths. Finally, we demonstrate that other types of biophysical cues that have an impact on cell orientation, such as directional fluid flow, give rise to the same cell stream pattern. These results demonstrate that external physical factors that exert subcellularscale physical constraints on individual cells within an unconfined monolayer can drive largescale collective movement patterns. 


\section{Results and Discussion}

\section{Endothelial cells on microgrooved substrates migrate with a periodic pattern of anti-} parallel cell streams

The movement of monolayers of human umbilical vein endothelial cells (HUVECs) was monitored on polydimethylsiloxane (PDMS) microstructured substrates composed of arrays of parallel grooves of width (w), spacing (s), and depth (d) of $5 \mu \mathrm{m}$, uniformly coated with fibronectin (Fig. 1a). Flat PDMS substrates served as controls. In line with previous reports ${ }^{11-}$ ${ }^{13}$, endothelial cells on the microgrooved substrates aligned and elongated in the groove direction (labeled as the $x$-axis) (Fig. 1a). At confluence, HUVEC nuclei were stained with the live-cell dye Hoechst and recorded for $24 \mathrm{~h}$ (Supplementary Video 1). While nuclei on the flat control surfaces moved in all directions with swirling-like trajectories, cell movement on the microgrooved substrates was constrained along the groove direction (Fig. 1b and Supplementary Video 1), leading to highly directional migration (Fig. 1c). A striking observation was the presence of a specific pattern of movement characterized by periodic streams of cells moving in opposite directions (i.e. anti-parallel) along the groove axis with a typical stream width of 100-150 $\mu \mathrm{m}$ (Fig. 1b), considerably greater than either the groove (5 $\mu \mathrm{m})$ or cell $(\sim 30 \mu \mathrm{m})$ size. This finding suggests that subcellular-scale physical constraints have wide-ranging ramifications on the organization and migration dynamics at the scale of the entire monolayer. Interestingly, the positions of the anti-parallel cell corridors remained relatively constant in time (see the cumulative trajectories, Fig. $1 \mathrm{~b}$ or the averaged $x$-velocity fields, Supplementary Fig. 1 and Video 2).

The extent of cell-cell coordination was assessed by comparing the orientation angle of the displacement of each cell at a given time to those of its neighbors within a distance of $100 \mu \mathrm{m}$ ( similar to Hayer et al. ${ }^{14}$ ). On flat surfaces, only local domains of aligned and coordinated 
movement were observed (Fig. 1b,d), similar to other studies on endothelial cells ${ }^{7,14,15}$ and reminiscent of nematic domains described for other cellular systems ${ }^{3,16,17}$. In contrast, on grooved substrates, a high degree of cellular coordination was observed within the cell streams, leading to overall higher and longer-range coordination compared to flat surfaces (Fig. 1e). Cell movement within confluent monolayers involves cell-cell rearrangements, creating cell-scale shearing. The high levels of cell-cell coordination in the cores of the streams led to small $\mathrm{x}$-direction cellular shear rates there, whereas high shear rates were generated locally at the stream borders due to the anti-parallel movement (Fig. 1f). Consequently, the average monolayer $\mathrm{x}$-direction shear rate and the average shear gradient in the y-direction were significantly higher on microgrooved substrates than on flat substrates (Fig. 1g). Shear forces on the endothelial apical surface due to blood flow is known to trigger myriad signaling events that exquisitely modulate vascular structure and function ${ }^{18-20}$; however, the shear rates in that case are on the order of $100-1,000 \mathrm{~s}^{-1}$, several orders of magnitude larger than the cell-scale shear rates reported here. It remains unclear if the shear rates and shear gradients induced by the cell stream pattern have significant functional ramifications. If so, potentially interesting targets might be the remodeling of cell-cell junctions and the potential impact on endothelial permeability and barrier function.

\section{The spatial features of the cell streams are modulated primarily by groove depth}

We then investigated the influence of groove dimensions on the cell streams. Reducing groove depth from 5 to $1 \mu \mathrm{m}$ while maintaining groove width and spacing constant $(5 \mu \mathrm{m})$ led to a wider range of cell orientation angles relative to the groove axis (Fig. 2a) and wider but shorter anti-parallel cell streams with more poorly defined borders (Fig. 2a,b). To quantify these observations, we computed the time- and x-averaged cell velocity as a function of the cross-stream coordinate y $\left(\bar{v}_{x}(y)\right.$; Fig. $\left.2 \mathrm{c}\right)$. Because of the anti-parallel nature of the streams, $\bar{v}_{x}(y)$ exhibits directional oscillations around zero. Reducing the groove depth led to less 
pronounced oscillations, indicating a weakening of the stream structure (Fig. 2c). As expected, the oscillations were essentially absent on the flat surfaces, reflecting the absence of cell streams. The median stream width, computed as the y-distance between zero crossings in the oscillations, increased from $\sim 100 \mu \mathrm{m}$ to $\sim 150 \mu \mathrm{m}$ as groove depth decreased from 5 to 1 $\mu \mathrm{m}$ (Fig. 2d). To quantify the length of the streams and their x-direction persistence, we considered the length over which the Pearson correlation coefficient between increasingly distant columns of the $x$-velocity field decreased from its original value of 1 to 0.2 . Stream length on $1 \mu \mathrm{m}$-deep grooves was $\sim 300 \mu \mathrm{m}$ and increased to $\sim 450 \mu \mathrm{m}$ on $5 \mu \mathrm{m}$-deep grooves (Fig. 2e). Interestingly, for a given groove depth, the stream length and width were markedly less sensitive to groove width and spacing than to groove depth (Supplementary Fig. 2). These findings suggest that the progressively higher constraint on the orientation of cell movement imposed by increased groove depth correlates with the transition from local regions of coordinated movement seen on flat surfaces to the longer-range interactions that lead to regular and persistent cell streams oriented in the direction of the grooves.

\section{The emergence of cell streams is dependent on cell-cell contacts}

Emergence of collective patterns of movement often requires cell-cell communication through cell-cell junctions that mature with increased cell density ${ }^{5,21}$. To elucidate the potential role of cell-cell contacts in the establishment of the anti-parallel streams, nuclear movement on $5 \mu \mathrm{m}$ deep grooves was recorded for different cell densities ranging from isolated (individual) endothelial cells to confluent monolayers. Individual cells traced highly linear trajectories in the groove direction with no bias between left-right or right-left movement (Supplementary Fig. 3). The cell streams developed over time as the cells proliferated and cell density increased, with faster establishment of clearly defined and stable streams for high-density monolayers (Supplementary Fig. 3 and Supplementary Video 3). These observations point towards an important role for cell-cell contacts in the establishment of the stream pattern. To 
further test this notion, we treated the cells with the calcium chelator ethylenediaminetetraacetic acid (EDTA; 2 and $5 \mathrm{mM}$ ) to disrupt cell-cell junctions during the recording time on $5 \mu \mathrm{m}$-deep grooves. Immunofluorescence staining confirmed that EDTA treatment strongly diminished the junctional recruitment of VE-cadherin (Supplementary Fig. 3). Importantly, EDTA treatment disrupted the stream pattern in a dose-dependent manner (Fig. 3a). Consistent with this observation, the amplitudes of the $\bar{v}_{x}$ oscillations were diminished (Fig. 3b,c). As confirmed by the increased ratio of $x$ - to $y$-displacement relative to the untreated monolayer (Fig. 3d), EDTA-treated cells exhibited more straight trajectories along the groove axis (Fig. 3a), a behavior similar to that of individual cells on grooves (cf: Supplementary Fig. 3). EDTA treatment significantly decreased cell-cell coordination (Fig. 3e), resulting in cells moving independently of neighboring cells and thus exhibiting "individual-like" behavior even though they remained in a monolayer. These results show that the loss of cell-cell contacts and coordination in endothelial monolayers on microgrooved substrates leads to disruption of the cell streams, indicating that this migration pattern is a form of organized collective motion that requires cell-cell communication.

\section{Active fluid modeling predicts the emergence of anti-parallel cell streams}

To gain insight into the physical basis for the emergence of endothelial cell streams, we developed an active fluid model inspired by that proposed by Duclos et al. ${ }^{4}$ who had described the emergence of global directional motion in monolayers confined within adhesive stripes. The model details are provided in the Supplementary Note. Briefly, our model includes two distinctive features that are specific to our system: 1) an energetic constraint on cell directionality to model the effect of substrate grooves, and 2) accounting for cell activity by including not only cell contractility but also individual cell migration. We model the cell sheet as an active fluid with cells treated as nematic active particles characterized by their orientation angle $\theta$ with respect to the groove direction (the $x$-axis). Active fluid theory 
includes constitutive relations that link the stress to the cell orientation angle, a continuity equation, and a force balance equation. Moreover, the system seeks to minimize its total free energy:

$$
\mathcal{F}=\int\left(\frac{K_{1}}{2}(\nabla \cdot \boldsymbol{p})^{2}+\frac{K_{3}}{2}(\nabla \times \boldsymbol{p})^{2}+\frac{\alpha}{2} p_{y}^{2}\right)
$$

where $\boldsymbol{p}=(\cos \theta, \sin \theta)$ is the unit director field, $\mathrm{K}_{1}$ and $\mathrm{K}_{3}$ are the splay and bend Frank constants, respectively. The two first terms are the classical terms representing energetic costs of variations in the orientation field, and the third term is an additional contribution arising from the energetic cost of any cell misalignment with the grooves, characterized by the parameter labeled $\alpha$ (with units of energy per unit surface). By using the Einstein relation, this coefficient can be estimated as $\alpha \approx \xi_{\mathrm{x}} D_{\mathrm{t}} / \sigma_{\theta}^{2}$, where $\xi_{\mathrm{x}}$ is the substrate friction coefficient, $D_{\mathrm{t}}$ the translational diffusion, and $\sigma_{\theta}$ the standard deviation of cell orientation angles, which is determined experimentally. The parameter $\alpha$ can thus be determined for any groove geometry and provides a useful, condensed description of the strength of cell alignment imposed by different types of mechanical cues.

Actomyosin activity is accounted for through a contractile force dipole acting on each cell $\zeta \Delta \mu$, where $\Delta \mu$ is the free energy produced by nutrient consumption and $\zeta$ is the reactive coefficient as defined in active gel theory ${ }^{22}$, as well as a net force between each cell and the substrate of typical magnitude $\xi_{x} v_{0}$, where $v_{0}$ is the typical speed of single cell migration.

Solving the model equations and calculating the full velocity profile in the $2 \mathrm{D}$ cell sheet reveals that the hydrodynamic profile that satisfies continuity is formed by alternating streams (Fig. 4a), a prediction that resembles the experimental velocity profile. The free energy is minimized for a stream width $W$ of the order of

$$
W^{2} \approx \frac{2 \pi^{2}(1+v) \gamma(-\zeta \Delta \mu)}{2 \alpha \xi_{x}}
$$


where $\eta$ and $\gamma$ are the shear and rotational viscosities, respectively, arising from both cell mechanical properties and cell-cell interactions. Typical order-of-magnitude parameter values (see table in Supplementary Note) lead to $W$ on the order of $100-200 \mu \mathrm{m}$, an estimate consistent with experimental observations (cf: Fig. 2d). Moreover, Eq. (3) yields the dependence $W \sim \alpha^{-1 / 2} \sim \sigma_{\theta}$. This predicted dependence is in excellent agreement with experimental observations (Fig.4c).

Rotational diffusion promotes cell misalignment with its neighbors, which leads to a persistence length of cell orientation that may correspond to the observed length of the streams. Two consecutive cells along a stream correspond to a step in a random walk. The persistence length of a stream $L$ can be predicted as the length along the $x$-axis for which the typical offset of the random walk along the $y$-axis is equal to the cell size, which leads to

$$
L \approx \frac{a}{\sigma_{\theta}^{2}}
$$

where $a \approx 90 \mu \mathrm{m}$ is the cell size (typical value on the microgroove surface). Measured standard deviations are of the order of $35^{\circ}-40^{\circ}$, i.e., $\sigma_{\theta} \approx 0.6$ to $0.7 \mathrm{rad}$, for which Eq. (4) yields estimates of the stream persistence length $L \approx 200$ to $280 \mu \mathrm{m}$, broadly in agreement with the experimental observations (cf: Fig. 2e). Moreover, the predicted dependence $L \propto$ $\sigma_{\theta}^{-2}$ provides an excellent description of the experimental behavior (Fig.4b).

In concert with the experimental observations, the modeling results indicate that the topographic substrates used here constitute a system which differs from both locally confined and free-boundary cellular monolayers, and where the collective behavior is driven not by local boundary effects as in other systems $s^{3-5,16,17}$ but rather by cell activity and biased cellular orientation due to local physical constraints. In addition, while topographic defect dynamics have been shown to be central in collective patterns of movement in many biological 
systems $^{16,17,23}$, this is not the case here because defects are expected to be essentially absent in our system due to the homogeneous and permanent cell alignment provided by the grooves.

\section{External shear flow also induces anti-parallel cell streams}

The model described above successfully captures the emergence of the experimentally observed cell streams and predicts that stream width and length are set by the proclivity for the cells to adopt a preferential orientation. An important consequence of this conclusion is that any source of constraint on cell orientation, and not only the anisotropic substrate topography studied thus far, would be expected to generate the periodic anti-parallel stream pattern. As a proof of principle, we recorded the collective migration of HUVEC monolayers on a flat surface subjected in a parallel plate microfluidic flow chamber to steady unidirectional (x-direction) flow (shear stress of $2 \mathrm{~Pa}$ for $24 \mathrm{~h}$ ). Anti-parallel cell streams similar to those observed on grooved substrates were indeed observed along the flow axis direction (Fig. 4c). Remarkably, the relationship between the stream dimensions (width and length) and the cellular orientation variance follows the same scaling law predicted by our model for the topography-induced streams (red squares in Fig. 4b). We note that the cell streams induced by external flow are often less oriented along the flow axis than those induced by substrate topography. Furthermore, the streams in the direction of the applied flow tended to be associated with higher migration velocities (by $20 \%$ ) than the counter-flow streams. These differences can be attributed to the different nature of the orienting cue: in contrast to the basal grooves that do not possess a directional bias along the groove axis and that exert their effect directly on the cells' focal adhesions and cytoskeletal organization ${ }^{11,13}$, the apical flow has intrinsic directionality and appears to have a more subtle and indirect impact on cell morphology as evidenced by the wider range of possible cell orientations (Fig. 4b). Nevertheless, the present observations suggest that the periodic anti-parallel cell streams 
described here may be a more universal pattern of collective movement that arises when unconfined cellular monolayers are subjected to externally orienting biophysical cues.

In vivo, endothelial collective migration occurs in geometrically unconfined monolayers but under the combined effects of anisotropic guidance constraints imposed by the complex structure of the underlying basement membrane, a scenario that our microgrooved substrates aimed to emulate, and directional shear forces due to luminal or interstitial blood flow. The periodic anti-parallel cell streams discovered here in vitro constitute a novel mode of collective migration that emerges when unconfined cellular monolayers are subjected to externally orienting biophysical cues. Whether these streams are also present in vivo remains unknown and is experimentally quite challenging to establish. However, if the present results apply to the in vivo situation, then the findings on the impact of groove depth on the cell streams would translate into an increased likelihood of observing these streams on thicker extracellular matrix fibers. Because loosening of arterial endothelial cell-cell junctions has been implicated in enhanced macromolecular permeability and thus in the development and progression of atherosclerotic lesions ${ }^{24,25}$, the present finding that intact cell-cell junctions are needed for establishing the anti-parallel cell stream pattern would lead us to expect this pattern to be largely absent in arterial zones prone to the development of atherosclerosis. Finally, in future studies, it would be interesting to establish if this migration pattern is also observed in other cell types and to explore its potential ramifications for a variety of important dynamic processes such as wound closure and maintenance of cellular barrier function. 


\section{Figures and legends}

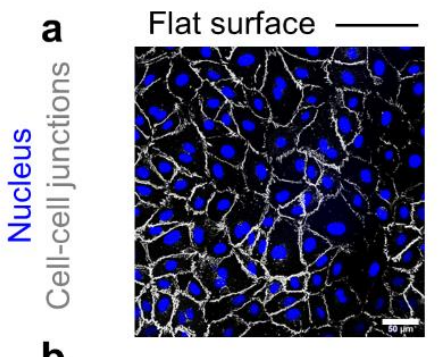

b

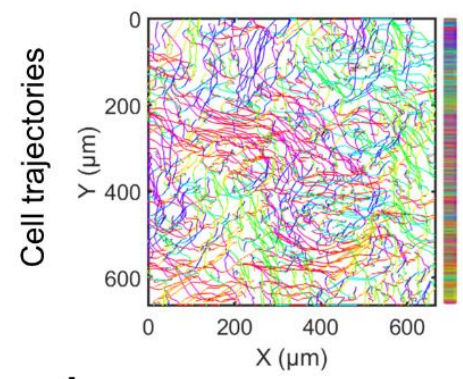

d
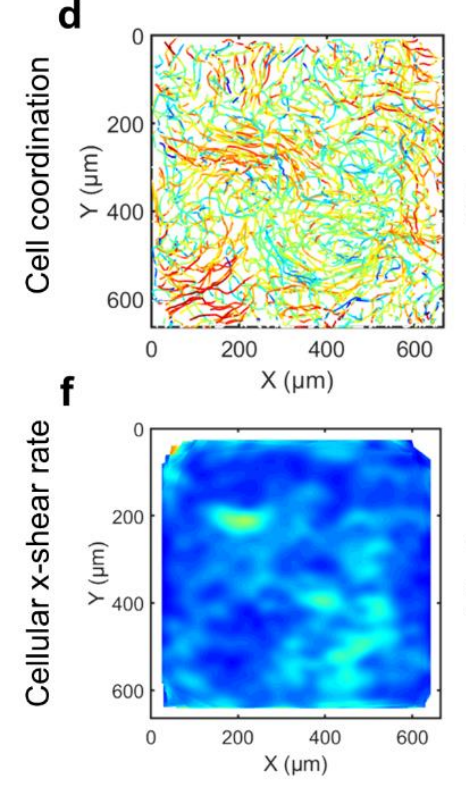
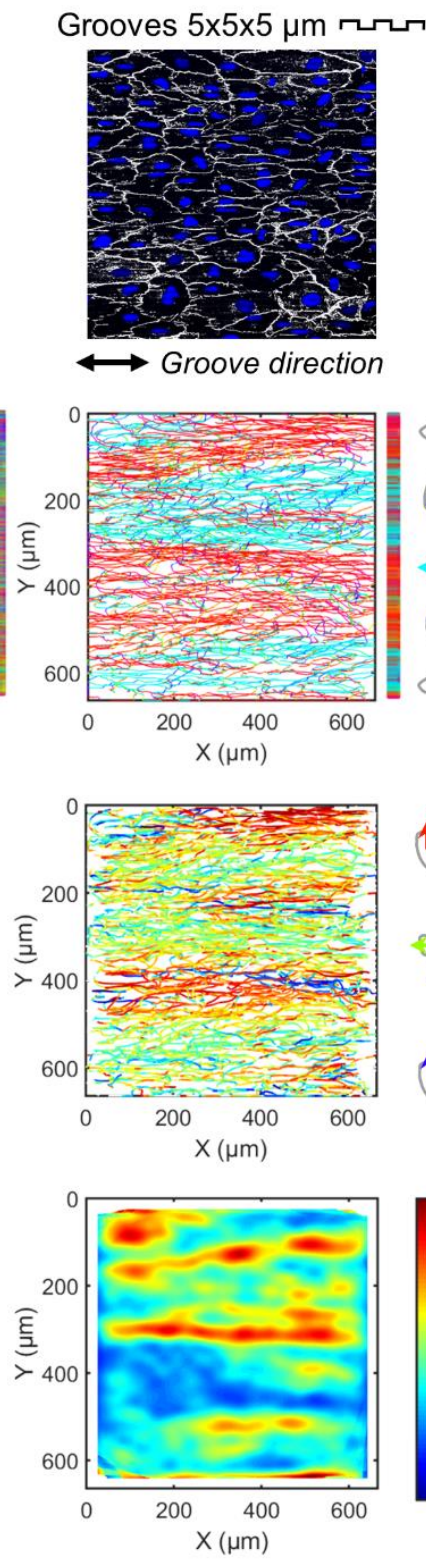

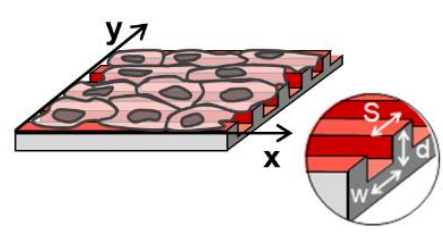

C
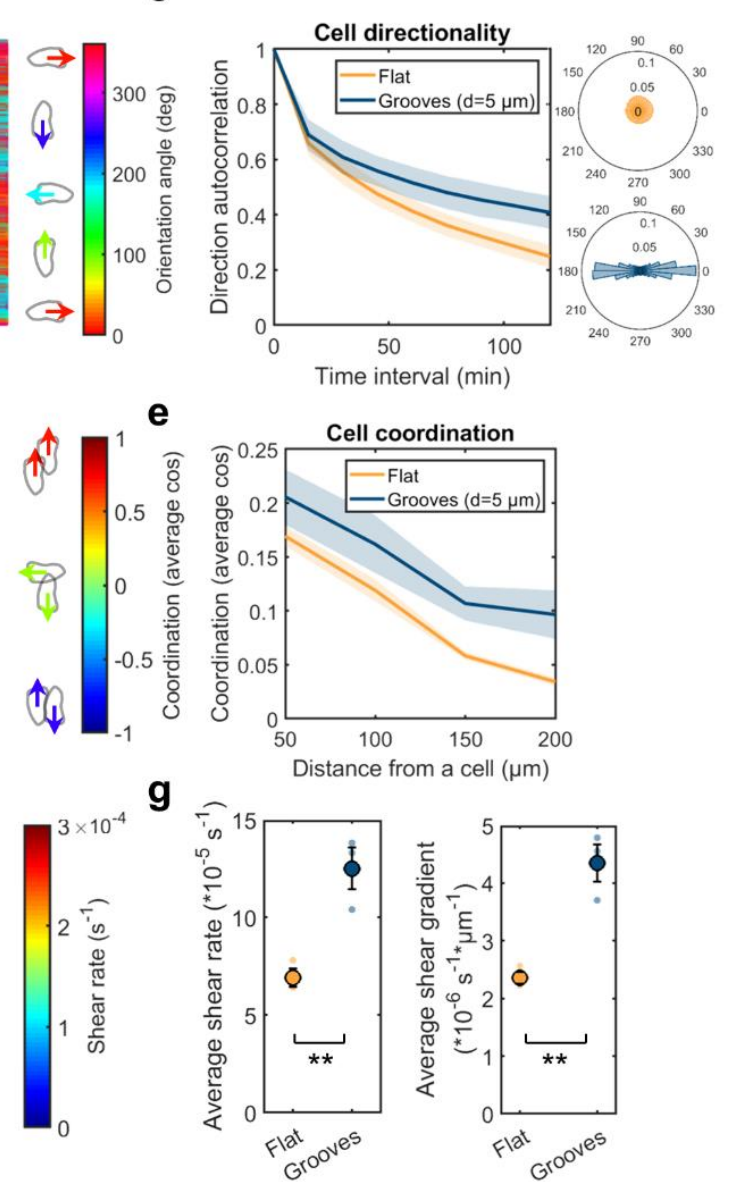

g

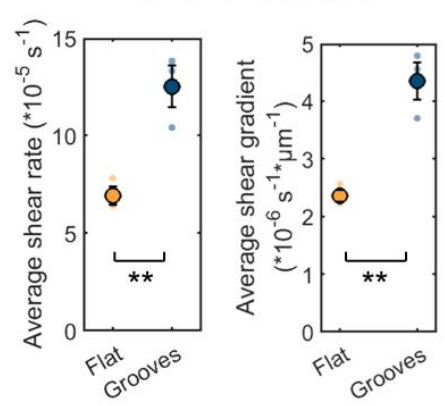

Figure 1: Endothelial cells on grooved substrates migrate with a periodic pattern of anti-parallel cell streams. a, Monolayers of endothelial cells (HUVECs) on a control flat substrate or substrate with grooves of width, spacing, and depth of $5 \mu \mathrm{m}$, immunostained for VE-cadherin (cell-cell junctions, white) and DAPI (nucleus, blue). Scale bar, $50 \mu \mathrm{m}$ and double-headed black arrow indicates groove direction. The grooved substrate is shown schematically on the right. $\mathbf{b}$, Accumulated cell trajectories after $24 \mathrm{~h}$ of migration color-coded for the orientation of each displacement vector. Vertical bars to the right of the trajectory maps represent the projected trajectories on the y-axis for better visualization of the cell streams. c, Direction autocorrelation analysis: the cosine of the angular difference between increasingly more distant vectors within the trajectory is computed and averaged for all cells (left). Polar histograms on the right show the distribution of displacement orientation angles. d, Cell trajectories color-coded for cell-cell coordination, defined as the cosine of the angular difference between a cell and its neighbors within a $100 \mu \mathrm{m}$ radius. Values of 1 and -1 indicate parallel and anti-parallel displacements, respectively. e, Evolution of the coordination parameter with increasingly distant neighbors. f, Heatmaps of cellular x-direction shear rate, extracted from the $\mathrm{x}$ - 
velocity field. g, Average x-direction shear rate and shear gradient in the y-direction. Dots represent individual experiments. Unpaired t-test (** $\mathrm{p}<0.001)$. For all graphs, $\mathrm{n}=3$ independent experiments and error bars represent SEM (standard error of the mean).
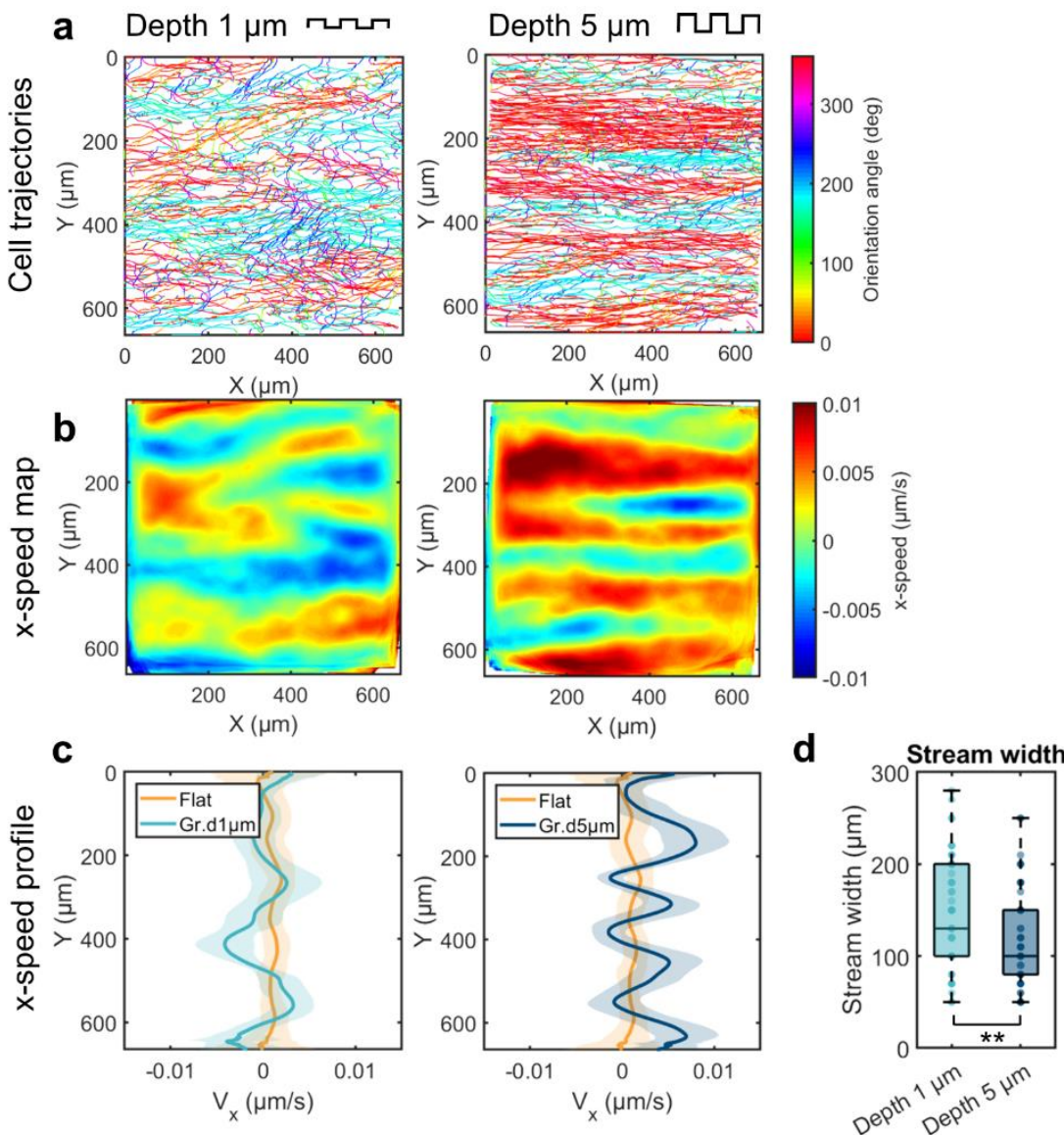

e
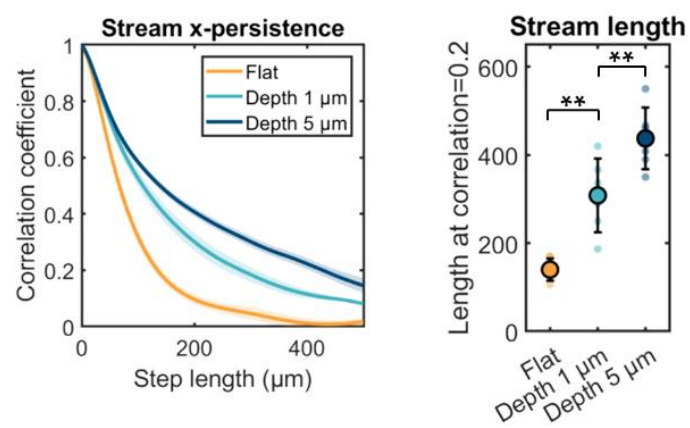

Figure 2: The spatial features of the streams are modulated by the groove depth. a, Cell trajectories after $24 \mathrm{~h}$ of migration on grooves of width and spacing of $5 \mu \mathrm{m}$ and depth of either $1 \mu \mathrm{m}$ (left) or $5 \mu \mathrm{m}$ (right) color-coded for orientation of displacement vectors. b, Heatmaps of the $\mathrm{x}$ - speed $\mathrm{V}_{\mathrm{x}}$, averaged in time. c, Corresponding $\mathrm{V}_{\mathrm{x}}$ profiles along the $\mathrm{y}$-axis averaged over time. Error bars represent standard deviations. d, The width of the streams was quantified as the average width of the peaks (average distance between zero crossings) in the $\mathrm{V}_{\mathrm{x}}$ profiles. Non parametric t-test $(* *, \mathrm{p}<$ 0.001) $\mathrm{n}=61$ streams (depth $1 \mu \mathrm{m}$ ), $\mathrm{n}=76$ streams (depth $5 \mu \mathrm{m}$ ) from 6 independent experiments. $\mathbf{e}$, Left: the stream persistence along the $\mathrm{x}$-axis was estimated by calculating the Pearson correlation coefficient between increasingly distant columns of the $V_{x}$ matrix. Error bars represent SEM. Right: the length of the streams was subsequently estimated as the distance corresponding to a correlation coefficient of 0.2. $\mathrm{n}=6$ independent experiments, one-way ANOVA, Tukey's post-test $(* *, \mathrm{p}<0.001)$. Error bars represent standard deviations. 

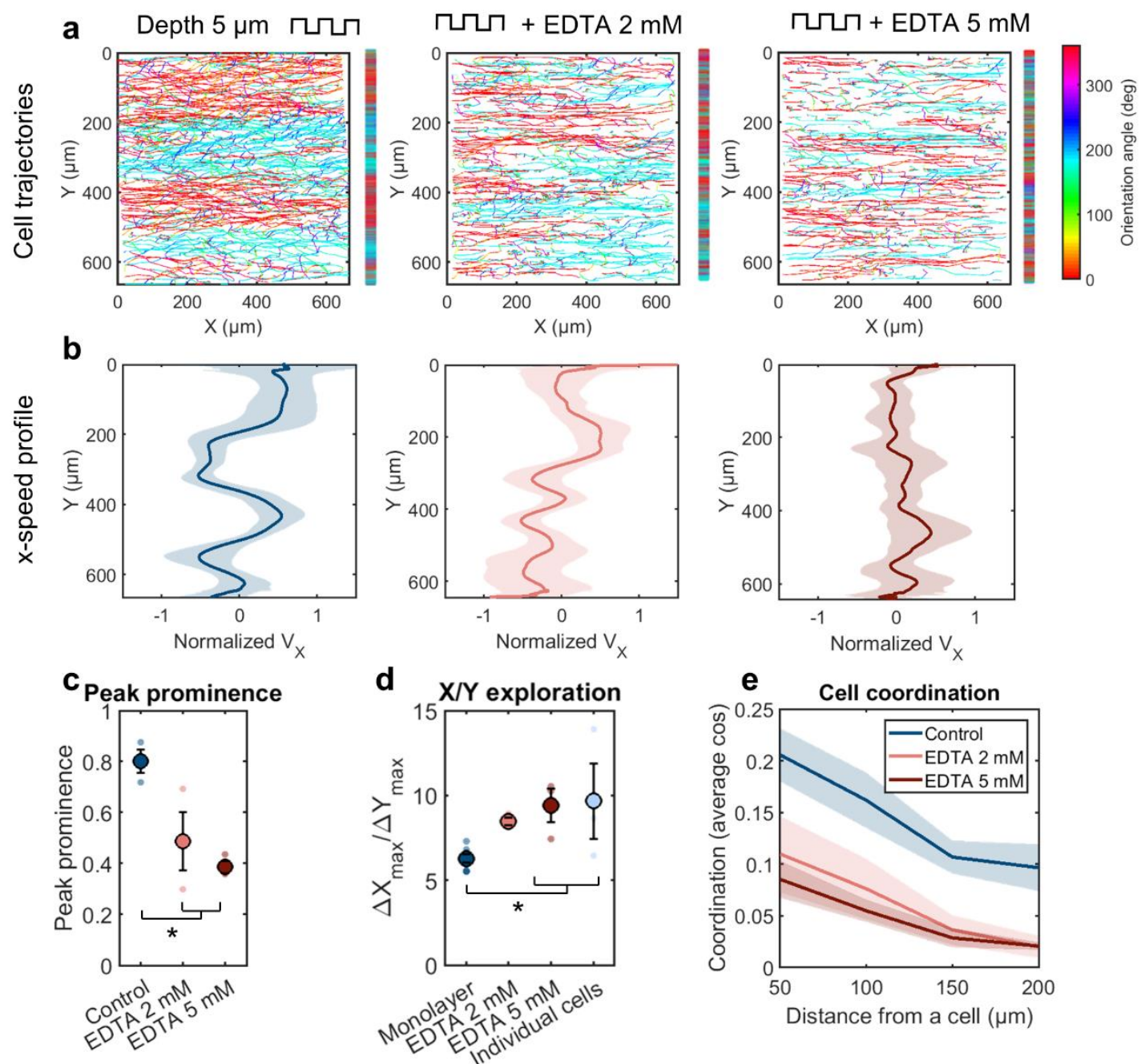

Figure 3: The emergence of cell streams is dependent on cell-cell contacts. a, Cell trajectories after $24 \mathrm{~h}$ of migration on grooves of width, spacing, and depth of $5 \mu \mathrm{m}$ with and without EDTA treatment. Trajectories are color-coded for orientation of displacement vectors. Vertical bars represent the projected trajectories on the $\mathrm{y}$-axis. $\mathbf{b}$, Corresponding $\mathrm{V}_{\mathrm{x}}$ velocity profiles (normalized by the mean speed) along the y-axis averaged over time. Error bars represent standard deviations. c, Peak prominence of $\mathrm{V}_{\mathrm{x}}$ profiles (defined as the relative peak height compared to the surrounding baseline). $\mathrm{n}=3$ independent experiments, one-way ANOVA, Tukey's post-test $(*, \mathrm{p}<0.01)$. d, Ratio between $\mathrm{x}$ and y-displacement (calculated as $|X \max -X \min | /|Y \max -Y \min |$ for each trajectory). The value for individual cells is shown for comparison. Monolayer $n=8$ independent experiments, EDTA and individual cells $n=3$ independent experiments. One-way ANOVA, Fishers's post-test $(*, p<0.01)$. e, Evolution of the cell coordination parameter with increasingly distant neighbors. Control $n=6$ independent experiments, EDTA $n=3$ independent experiments. For all graphs, error bars represent SEM. 

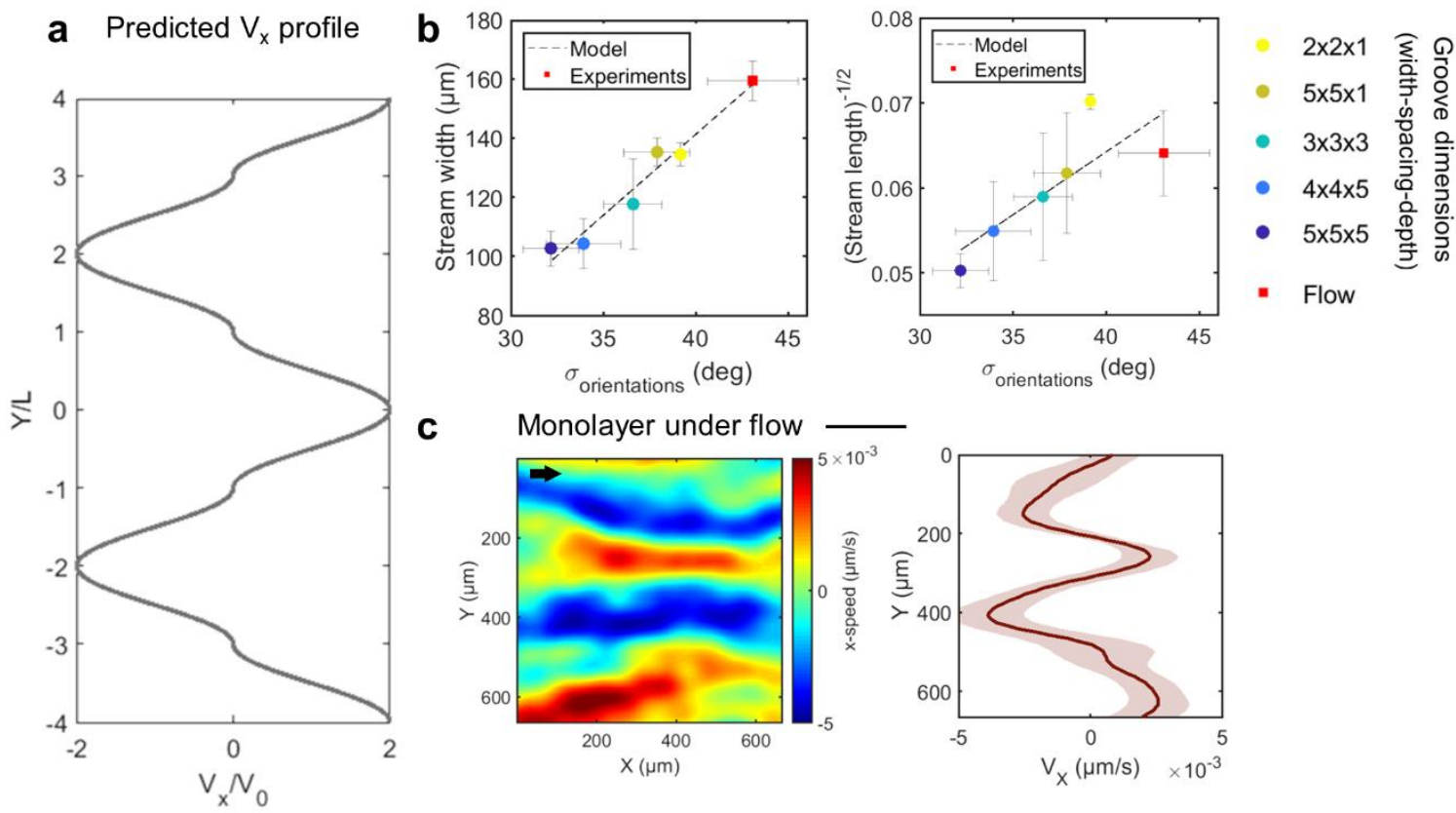

C Monolayer under flow

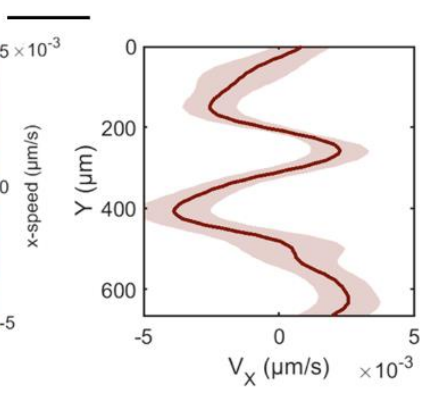

Figure 4: Modeling the emergence of cell streams. a, $V_{X}$ profile predicted by the model. $\mathbf{b}$, Relationship between the spatial features of the streams and the standard deviation of the displacement vector orientation angles. Dots represent experimental points and dashed lines the linear fit predicted by the model. Error bars represent SEM. c, $\mathrm{V}_{\mathrm{X}}$ heatmap and corresponding $\mathrm{V}_{\mathrm{X}}$ profile of HUVEC monolayer on a flat surface subjected to a laminar shear stress of $2 \mathrm{~Pa}$ for $24 \mathrm{~h}$. Black arrow indicates the flow direction. 


\section{Materials \& Methods}

\section{Fabrication of microgrooved substrates}

The original microstructured silicon wafer was fabricated using classical photolithography procedures by UV illumination (MJB4 Mask Aligner, $23 \mathrm{~mW} / \mathrm{cm}^{2}$ power lamp, SUSS MicroTec, Germany) of a layer of SU8-2010 (MicroChem, USA) through a hard chromium mask. After exposure to vapor of trichloro $(1 \mathrm{H}, 1 \mathrm{H}, 2 \mathrm{H}, 2 \mathrm{H}$-perfluorooctyl)silane (Sigma) for 20 min, the silicon wafer was used to create polydimethylsiloxane (PDMS Sylgard 184, Sigma Aldrich, ratio 1:10) replicates. To create the final coverslip on which the cells were cultured, liquid PDMS was spin coated at $1500 \mathrm{rpm}$ for $30 \mathrm{~s}$ on the PDMS mold. Before reticulation overnight at $70^{\circ} \mathrm{C}$, a glass coverslip was placed on top of the PDMS layer. After reticulation, the glass coverslip attached to the microstructured PDMS layer was gently demolded with a scalpel and isopropanol to facilitate detachment. Microstructured coverslips were then sonicated for $10 \mathrm{~min}$ in ethanol for cleaning and finally rinsed in water.

\section{Cell culture}

Prior to cell seeding and after a $30 \mathrm{~s}$ plasma treatment, the microgrooved substrates were incubated for $1 \mathrm{~h}$ with $50 \mu \mathrm{g} / \mathrm{ml}$ fibronectin solution (Sigma) at room temperature. Human umbilical vein endothelial cells (HUVECs, Lonza) in passages 4-8 were cultured in EGM2MV medium (Lonza) at $37^{\circ} \mathrm{C}$ in a humidified atmosphere of $95 \%$ air and $5 \% \mathrm{CO}_{2}$. At confluence, cells were detached with trypsin (Gibco, Thermo Fisher Scientific) and seeded onto either control PDMS substrates or microgrooved coverslips at densities of 30,000-50,000 cells $/ \mathrm{cm}^{2}$. After $24 \mathrm{~h}$ of culture and prior to imaging, cells were incubated for $3 \mathrm{~min}$ with Hoechst 33342 (Thermo Fisher Scientific, dilution 1/10,000) for live-cell nuclear fluorescence labeling. 
For the ethylenediaminetetraacetic acid (EDTA, Sigma) experiments, cells were incubated after $24 \mathrm{~h}$ of culture with 2 or $5 \mathrm{mM}$ EDTA in culture medium for the duration of the imaging protocol. Immunostaining against VE-cadherin was performed at the end of the imaging to check for EDTA treatment efficacy.

\section{Immunostaining}

Culture coverslips were fixed with $4 \%$ paraformaldehyde (Thermo Fisher) in PBS for 15 min. After $1 \mathrm{~h}$ in a blocking solution containing $0.25 \%$ Triton and $2 \%$ bovine serum albumin (BSA), cultures were incubated for $1 \mathrm{~h}$ at room temperature with rabbit anti-VE-cadherin primary antibody (ab33168, Abcam) diluted $1 / 400$ in a solution containing $0.25 \%$ Triton and $1 \%$ bovine serum albumin (BSA). Coverslips were washed three times with PBS and incubated for $1 \mathrm{~h}$ at room temperature with Alexa Fluor 555-conjugated donkey anti-rabbit antibody (ab150074, Abcam) and DAPI.

\section{Time-lapse microscopy}

Live recordings of HUVEC monolayers were performed after $24 \mathrm{~h}$ of culture with an automated inverted microscope (Nikon Eclipse Ti) equipped with temperature and $\mathrm{CO}_{2}$ regulation and controlled by the NIS software (Nikon). Images were acquired with a 20X objective (Nikon Plan Fluor NA=0.5) for $24 \mathrm{~h}$ at $15 \mathrm{~min}$ intervals. Three fields of views (FOVs) were randomly chosen from each experiment for the control (flat) and microgrooved regions of the coverslip, and 3 to 6 separate experiments were conducted for each experimental condition.

\section{Flow experiments}

For flow experiments on HUVECs, $1 \times 10^{5}$ cells $/ \mathrm{cm}^{2}$ were seeded in fibronectin-coated Ibidi slides ( $\mu$-Slide I 0.4 Luer, Ibidi, Biovalley, France). After 2 h, the parallel plate flow chamber 
was placed on the stage of an inverted microscope (Nikon Eclipse Ti) equipped with temperature and $\mathrm{CO}_{2}$ control and inserted into a recirculating flow loop to subject the cells to steady unidirectional shear stress. Continuous flow of cell culture medium (EGM2-MV, Lonza) was generated by a peristaltic pump (Cole-Parmer, USA). Flow exiting the pump passed through a pulse dampener (Cole-Parmer, USA) to ensure flow steadiness before reaching the cell sample. After a gradual increase of shear stress from 0.5 to $2 \mathrm{~Pa}$ over a period of $1 \mathrm{~h}$, brightfield images were acquired using a 10X objective (Nikon Plan NA=0.25) for $24 \mathrm{~h}$ at $10 \mathrm{~min}$ intervals. Four independent experiments were analyzed.

\section{Image processing and data analysis}

Tracking of the nucleus from time-lapse acquisitions was performed semi-automatically using the MTrackJ plugin in ImageJ. Subsequent analyses were performed in Matlab (MathWorks) using custom-written scripts.

Directionality analysis. Cell directionality was assessed using the direction autocorrelation (DA) analysis, performed by the VBA Excel Macro provided by Gorelik et al. ${ }^{26}$ and based on the following equations:

$$
\begin{aligned}
& D A=\frac{1}{N-n+1} \sum_{i=0}^{N-n}\left(\vec{v}_{(i+n) \Delta t} \cdot \vec{v}_{i \Delta t}\right)=\frac{1}{N-n+1} \sum_{i=0}^{N-n}\left(\cos \left(\alpha_{(i+n) \Delta t}-\alpha_{i \Delta t}\right)\right) \\
& \langle D A\rangle_{C}=\sum_{j=1}^{j=C}(D A)_{j} \cdot N_{j} / \sum_{k=1}^{k=C} N_{k}
\end{aligned}
$$

where DA denotes the average direction autocorrelation coefficient for a given cell at step size $\mathrm{n}, \mathrm{N}$ represents the total number of displacements, $\Delta \mathrm{t}$ is the time interval between 2 points in the trajectory, $\alpha$ is the angle at each time point of the trajectory, and $\mathrm{C}$ is the total number of cells. 
Coordination analysis. Cell-cell coordination was calculated using a method similar to that described by Hayer et al. ${ }^{14}$ and using Eq. (3) below. More specifically, the coordination parameter $\mathrm{C}\left(\Delta_{\mathrm{R}}\right)$ was calculated as the mean cosine of the angle between the direction of one cell and that of all neighbors within a $100 \mu \mathrm{m}$-radius circle or $50 \mu \mathrm{m}$-wide circular rings located at different distances $\Delta_{\mathrm{R}}(0-50 \mu \mathrm{m}, 50-100 \mu \mathrm{m}, 100-150 \mu \mathrm{m}, 150-200 \mu \mathrm{m})$ from the considered cell. The coordination parameter is subsequently averaged for one cell along its trajectory and for all cells present in the FOV.

$\mathrm{C}\left(\Delta_{\mathrm{R}}\right)=\frac{1}{N} \sum_{i=1}^{N}\left(\frac{1}{T_{i}} \sum_{t=1}^{T_{i}}\left(\frac{1}{n_{i, t}} \sum_{j=1}^{n_{i, t}} \cos \left(\alpha_{i, t}-\alpha_{j, t}\right)\right)\right)$

where $\mathrm{N}$ is the total number of cells in the $\mathrm{FOV}, \mathrm{T}_{\mathrm{i}}$ is the total number of points in the trajectory of cell $\mathrm{i}, \mathrm{n}$ is the total number of neighbors around cell $\mathrm{i}$ in frame $\mathrm{t}$, and $\alpha_{\mathrm{i}, \mathrm{t}}$ is the angle of the displacement vector of cell $\mathrm{i}$ in frame $\mathrm{t}$.

Speed interpolation analysis. Continuous x-speed maps were generated using a custom MATLAB script that interpolates speed values on a regular grid from the discrete positions of the nucleus extracted from the tracking.

Cellular shear rate analysis. Cellular shear rate in the $\mathrm{x}$-direction $\left(S R_{x}\right)$ was computed from the interpolated speed matrix as $S R_{x}=d V_{x} / d y$. Similarly, cellular y-direction shear gradient $(\nabla \mathrm{SR} x)$ was calculated from the $S R_{x}$ matrix as $\nabla \mathrm{SR} \mathrm{R}_{x}=d S R_{x} / d y$

Flow experiment analysis. Particle image velocimetry (PIV) was performed on brightfield images from flow experiments using the PIVlab package in MATLAB in order to extract the $\mathrm{x}$-speed matrix. The window size was set to 100 pixels $=65 \mu \mathrm{m}$ with a 0.5 overlap for the first pass and 50 pixels $=32.5 \mu \mathrm{m}$ for the second pass. 


\section{Statistical analysis}

All analyses are based on 3 to 6 independent experiments using 3 different FOVs from each experiment and with each FOV containing approximately 200 to 300 cells. Statistical analyses were performed using the GraphPad Prism software. The Student t-test and the MannWhitney t-test were used to compare null hypotheses between two groups for normally and non-normally distributed data, respectively. Multiple groups with a normal distribution were compared by ANOVA, followed by Tukey's or Fisher's posthoc test. The number of data points for each experiment, the specific statistical tests, and the significance levels are noted in the corresponding figure legends. 


\section{Acknowledgments}

This work was supported in part by an endowment in Cardiovascular Bioengineering from the AXA Research Fund (to AIB) and a postdoctoral fellowship from the Lefoulon-Delalande Foundation (to CL).

\section{Author Contributions}

C.L. and A.I.B. designed the research. C.L. and T.L. performed the experiments, C.L., A.V. and AM.D. performed data analysis, and D.G-R. developed the theory. C.L., D.G-R. and A.I.B. wrote the manuscript.

\section{Competing Interests statement}

The authors declare no competing interests.

\section{Data Availability}

The data that support the findings of this study are available from the corresponding author upon reasonable request. 


\section{References}

1. Friedl, P. \& Gilmour, D. Collective cell migration in morphogenesis, regeneration and cancer. Nat. Rev. Mol. Cell Biol. 10, (2009).

2. Doxzen, K. et al. Guidance of collective cell migration by substrate geometry. Integr. Biol. 5, (2013).

3. Duclos, G., Garcia, S., Yevick, H. G. \& Silberzan, P. Perfect nematic order in confined monolayers of spindle-shaped cells. Soft Matter 10, (2014).

4. Duclos, G. et al. Spontaneous shear flow in confined cellular nematics. Nat. Phys. 14, 728-732 (2018).

5. Vedula, S. R. K. et al. Emerging modes of collective cell migration induced by geometrical constraints. Proc. Natl. Acad. Sci. 109, (2012).

6. Szabó, A. et al. Collective cell motion in endothelial monolayers. Phys. Biol. 7, (2010).

7. Lin, S.-Z., Ye, S., Xu, G.-K., Li, B. \& Feng, X.-Q. Dynamic Migration Modes of Collective Cells. Biophys. J. 115, (2018).

8. Liliensiek, S. J., Nealey, P. \& Murphy, C. J. Characterization of endothelial basement membrane nanotopography in rhesus macaque as a guide for vessel tissue engineering. Tissue Eng. Part A 15, 2643-51 (2009).

9. Brody, S. et al. Characterizing Nanoscale Topography of the Aortic Heart Valve Basement Membrane for Tissue Engineering Heart Valve Scaffold Design. Tissue Eng. 12(2), 413-21 (2006).

10. Leclech, C., Natale, C. F. \& Barakat, A. I. The basement membrane as a structured 
surface - role in vascular health and disease. J. Cell Sci. 133, jcs239889-undefined (2020).

11. Antonini, S. et al. Sub-micron lateral topography affects endothelial migration by modulation of focal adhesion dynamics. Biomed. Mater. (2015) doi:10.1088/17486041/10/3/035010.

12. Stefopoulos, G., Giampietro, C., Falk, V., Poulikakos, D. \& Ferrari, A. Facile endothelium protection from TNF- $\alpha$ inflammatory insult with surface topography. Biomaterials 138, 131-141 (2017).

13. Natale, C. F., Lafaurie-Janvore, J., Ventre, M., Babataheri, A. \& Barakat, A. I. Focal adhesion clustering drives endothelial cell morphology on patterned surfaces. J. R. Soc. Interface 16, 20190263 (2019).

14. Hayer, A. et al. Engulfed cadherin fingers are polarized junctional structures between collectively migrating endothelial cells. Nat. Cell Biol. 18, 1311-1323 (2016).

15. Vitorino, P. \& Meyer, T. Modular control of endothelial sheet migration. Genes Dev. 22, (2008).

16. Duclos, G., Erlenkämper, C., Joanny, J.-F. \& Silberzan, P. Topological defects in confined populations of spindle-shaped cells. Nat. Phys. 13, (2017).

17. Kawaguchi, K., Kageyama, R. \& Sano, M. Topological defects control collective dynamics in neural progenitor cell cultures. Nature 545, (2017).

18. Chien, S. Mechanotransduction and endothelial cell homeostasis: The wisdom of the cell. in American Journal of Physiology - Heart and Circulatory Physiology vol. 292 (Am J Physiol Heart Circ Physiol, 2007). 
19. Hahn, C. \& Schwartz, M. A. Mechanotransduction in vascular physiology and atherogenesis. Nat. Rev. Mol. Cell Biol. 10, (2009).

20. Barakat, A. I. Blood flow and arterial endothelial dysfunction: Mechanisms and implications. Comptes Rendus Phys. 14, 479-496 (2013).

21. Garcia, S. et al. Physics of active jamming during collective cellular motion in a monolayer. Proc. Natl. Acad. Sci. 112, (2015).

22. Kruse, K., Joanny, J. F., Jülicher, F., Prost, J. \& Sekimoto, K. Generic theory of active polar gels: a paradigm for cytoskeletal dynamics. Eur. Phys. J. E 16, (2005).

23. Blanch-Mercader, C. et al. Turbulent Dynamics of Epithelial Cell Cultures. Phys. Rev. Lett. 120, (2018).

24. Stemerman, M. B. et al. Local variation in arterial wall permeability to low density lipoprotein in normal rabbit aorta. Arterioscler. An Off. J. Am. Hear. Assoc. Inc. 6, (1986).

25. Weinbaum, S., Tzeghai, G., Ganatos, P., Pfeffer, R. \& Chien, S. Effect of cell turnover and leaky junctions on arterial macromolecular transport. Am. J. Physiol. Circ. Physiol. 248, (1985).

26. Gorelik, R. \& Gautreau, A. Quantitative and unbiased analysis of directional persistence in cell migration. Nat. Protoc. 9, 1931-1943 (2014).

27. Voituriez, R., Joanny, J. F. \& Prost, J. Spontaneous flow transition in active polar gels. Europhys. Lett. 70, (2005). 